\title{
Intraocular lens power calculation for planned ametropia: a clinical study
}

\author{
JEFFREY S. HILLMAN \\ From St James's University Hospital, Leeds
}

SUMmARY A prospective series of 25 eyes received an intraocular lens (IOL) of power calculated for planned ametropia, by means of the formulae of R. D. Binkhorst, from data of axial length, corneal curvature, and postoperative anterior chamber depth. All the postoperative refractions were within the $\pm 2 \mathrm{D}$ range from the predicted refraction, confirming the clinical value of such calculation. A retrospective study of 100 eyes which had received a $+19 \mathrm{D}$ power Binkhorst IOL showed a wide range of change in refraction extending up to the $\pm 6 \mathrm{D}$ range, indicating that a 'standard' power IOL cannot be relied upon to reproduce the preoperative refraction. Calculation of IOL power from biometric data is essential when controlled postoperative ametropia is required.

The intraocular lens (IOL) offers the aphakic patient vision of normal quality which is superior to the magnified and distorted vision of aphakic spectacles and without the demand on manual dexterity of the contact lens. It is now generally accepted that the visual benefits of the IOL outweigh the risks of the additional intraocular manipulation at operation. ${ }^{1}$ The postoperative refraction is an important aspect of the optical correction, and a number of studies have demonstrated the unpredictable refractive outcome with a 'standard' power IOL and the control which can be exercised over the postoperative refraction by the calculation of IOL power. ${ }^{2-6}$

The refractive goal for any particular eye will depend on the preoperative refractions and visual acuities of the 2 eyes and on the philosophy of the surgeon. For most eyes, when the fellow eye is nearemmetropic or when a fellow ametropic eye has little vision and bilateral surgery is planned, the desirable postoperative refraction will be emmetropia or low myopia. In some cases, however, when the fellow eye has significant ametropia with good vision, the operated eye will require an IOL of power to give matching ametropia to avoid problems from anisometropia.

The power of IOL for ametropia may be determined by calculation from biometric data, but few results have been published, and one aim of this study was to assess the accuracy of such calculation. The second aim was to test the hypothesis that such

Correspondence to Mr J. S. Hillman, FRCS, Department of Ophthalmology, St. James's University Hospital, Leeds LS9 7TF. calculation may be unnecessary, as a 'standard' power IOL may be relied upon to reproduce postoperatively the preoperative basic refraction.

\section{Material and methods}

The first part of this study was performed prospectively on 25 eyes for which clinical considerations indicated an IOL of power to give postoperative ametropia to match the fellow eye. Calculation was made by the formulae of R. D. Binkhorst ${ }^{7}$ programmed into a computer for convenience, and the following formula gave the IOL power for a stated postoperative refraction:

$$
\begin{aligned}
& D= \frac{1336\left\{4 r-a-[v(4 r-a)+0 \cdot 003 a r] R_{s}\right\}}{(a-d)\left\{4 r-d-[v(4 r-d)+0 \cdot 003 d r] R_{s}\right\}} \\
& D= \text { IOL power in aqueous (dioptres). } \\
& r= \text { Corneal radius }(m m) . \\
& a= \text { Axial length (mm). } \\
& v= \text { Back vertex distance (metres). } \\
& R_{s}=\text { Spectacle refraction (dioptres). } \\
& d=\text { Postoperative anterior chamber depth plus corneal thickness } \\
&(\mathrm{mm}) .
\end{aligned}
$$

The IOL implanted was of the nearest whole dioptre power to that calculated, as the limitations of accuracy of biometry do not justify the use of IOL powers in fractions of a dioptre. ${ }^{8}$ The following formula was used to predict the postoperative refraction with the IOL power actually implanted:

$$
R_{s}=\frac{1336(4 r-a)-D(a-d)(4 r-d)}{1336[v(4 r-a)+0 \cdot 003 a r]-D(a-d)[v(4 r-d)+0 \cdot 003 d r]}
$$


Fig. 1 Comparison between the predicted postoperative refractions and the actual postoperative refractions (as spherical equivalent power) in 25 eyes which received IOLs calculated for planned ametropia. The oblique line indicates the points of achievement of the predicted refraction.

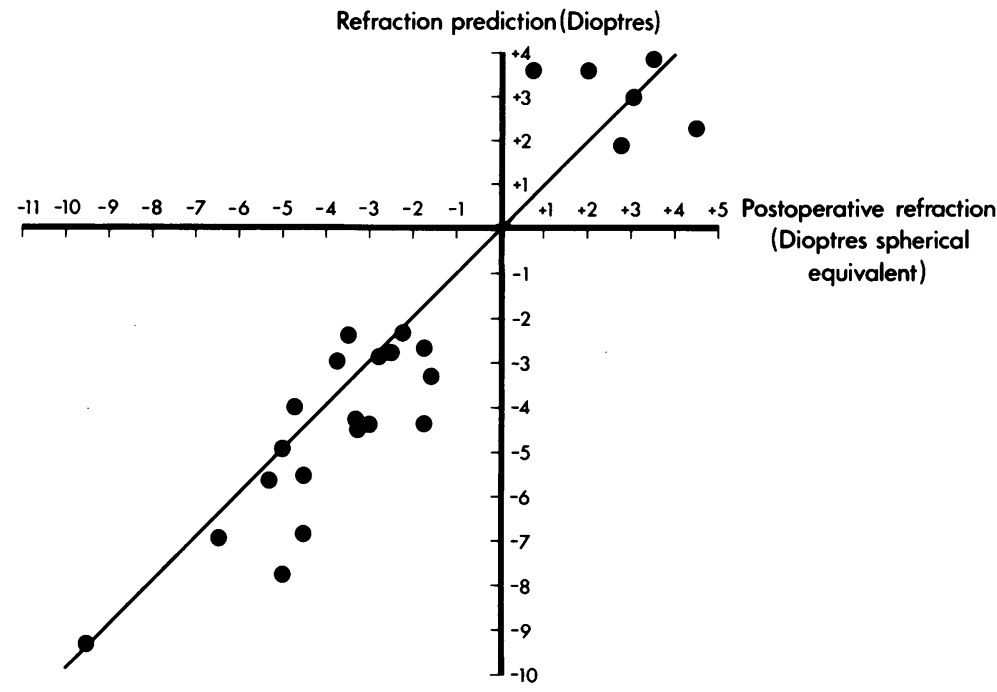

Axial length was measured by ultrasound with a Kretz 7200 MA ophthalmic A-scan with a $10 \mathrm{MHz}$ transducer used in a contact lens water bath containing 5\% methylcellulose. Measurements were taken from Polaroid photographs of axial scans on the assumption of a value of 1550 metres/second for the common speed of ultrasound in ocular tissues. A correction factor of $0.25 \mathrm{~mm}$ was added to the measured axial length to correct for the several factors which tend to give undermeasurement. ${ }^{8}$ Corneal curvature was taken as the average of measurements made in 2 meridia with a Haag-Streit keratometer. A value of $3.19 \mathrm{~mm}$ was assumed for the postoperative distance from the vertex of the cornea to the anterior vertex of the Binkhorst IOL.
Cataract extraction was performed personally by a microsurgical technique with general anaesthesia and hyperventilation. A limbal incision was made ab externo under a limbal-based conjunctival flap, and after a single peripheral iridectomy $\alpha$-chymotrypsin was instilled and the lens extracted by cryoprobe. The pupil was constricted with acetylcholine, and a Rayner-Binkhorst IOL was inserted into the pupil by the closed-chamber technique. A loose 10/0 safetysling suture was placed via the upper anterior loop of the IOL and the margin of the iridectomy at the junction of the middle and outer thirds of the iris. The wound was closed with interrupted virgin silk $8 / 0$ sutures, particular attention being paid to suture tension to minimise surgical astigmatism.
Fig. 2 Comparison between the predicted postoperative refractions and the actual postoperative refractions (as spherical power) in 25 eyes which received IOLs calculated for planned ametropia. The oblique line indicates the points of achievement of the predicted refraction.

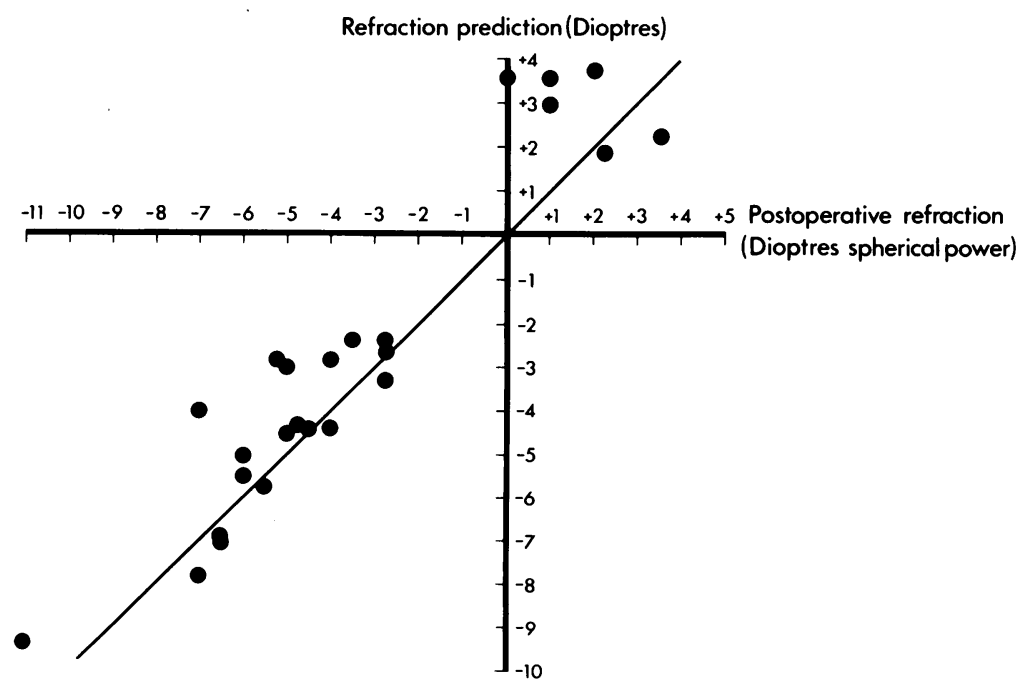


The postoperative refraction was recorded as spherical equivalent power between the 6th and 8th postoperative week when the first spectacles were prescribed and was compared with the predicted refraction.

The second part of the study to determine the effect on the refraction of implantation of a 'standard' +19 D power Binkhorst IOL was carried out retrospectively on 100 eyes which had received such an IOL and had served as a control population in a study of IOL power calculation for emmetropia. ${ }^{6}$ In order to minimise the effect of cataract-induced refraction change the basic preoperative refraction was taken as the prescription in the patient's spectacles at the time of first referral to the ophthalmic surgeon. In most cases these spectacles were dispensed from a refraction a year or more before referral. Such information was available for 96 of the 100 eyes, and this preoperative refraction was compared with the postoperative refraction between the 6th and 8th postoperative week.

\section{Results}

The differences between the predicted refractions and the postoperative refractions are presented graphically in Fig. 1 as spherical equivalent power and in Fig. 2 as spherical power (after transposition to plus cylinder form). The results are presented numerically in Table 1 . All the eyes were within the $\pm 2 \mathrm{D}$ range and $80 \%$ within the $\pm 1 \mathrm{D}$ range for spherical equivalent power and $92 \%$ and $80 \%$ respectively for spherical power. The mean differences between the predicted refraction and the postoperative refraction $( \pm$ SD) were $1.07( \pm 0.89) \mathrm{D}$ for spherical equivalent and $1 \cdot 15( \pm 0 \cdot 97) \mathrm{D}$ for spherical power. The results are better for the myopic eyes, but the hypermetropic series is only a small one. The

Table 1 The difference between the predicted and actual postoperative refractions as dioptres spherical equivalent power and spherical power in 25 eyes which received IOLs calculated for planned ametropia.

\begin{tabular}{clc}
\hline Dioptres & Spherical equivalent & Spherical power \\
\hline 0 to 0.99 & $60 \%$ & $56 \%$ \\
1.0 to 1.99 & $20 \%$ & $24 \%$ \\
2.0 to 2.99 & $20 \%$ & $12 \%$ \\
3.0 to 3.99 & - & $8 \%$ \\
\hline
\end{tabular}

Table 2 The powers of the IOLs in dioptres used in 25 eyes which received IOLs calculated for planned ametropia.

\begin{tabular}{lrrrrrrrrrrr}
\hline IOL power & 15 & 16 & 17 & 18 & 19 & 20 & 21 & 22 & 23 & 24 & 25 \\
\hline Number & 4 & 4 & 4 & 5 & 1 & 3 & 0 & 1 & 0 & 2 & 1 \\
\hline
\end{tabular}

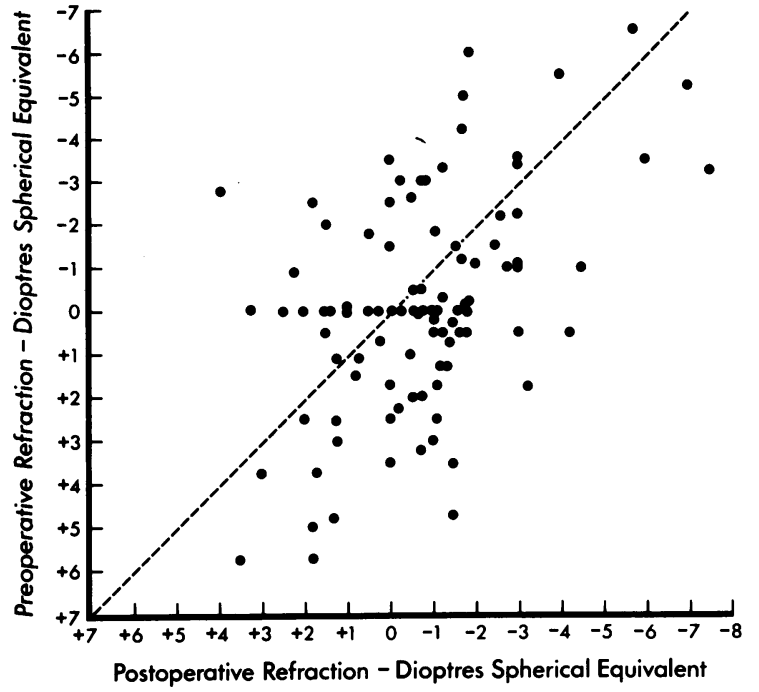

Fig. 3 Comparison between the preoperative and postoperative refractions (as spherical equivalent power) in 97 eyes which received a 'standard' +19 D Binkhorst IOL.

range of IOL powers calculated for these eyes is shown in Table 2 and is wide with a mean value $( \pm \mathrm{SD})$ of $18 \cdot 24( \pm 2 \cdot 86) \mathrm{D}$.

A comparison between the preoperative spherical equivalent refraction and the postoperative spherical equivalent refraction for the eyes which received a +19 D Binkhorst IOL is presented graphically in Fig. 3 and numerically in Table 3 . The mean change in refraction $( \pm$ SD $)$ was $1.99( \pm 1 \cdot 38) \mathrm{D}$, and only $78 \cdot 1 \%$ of the eyes were within the $\pm 2 \mathrm{D}$ range. $21.9 \%$ eyes had a greater change in refraction extending into the $\pm 6 \mathrm{D}$ range. The variation in refractive change with a 'standard' power IOL was so wide as to make the refractive outcome unpredictable with any degree of accuracy.

\section{Discussion}

The calculation of IOL power for planned postoperative ametropia from the formulae of R. D.

Table 3 The change in refraction (spherical equivalent power) following implantation of a 'standard' $+19 \mathrm{D}$ power Binkhorst IOL in 97 eyes.

\begin{tabular}{rr}
\hline Dioptres & $\%$ \\
\hline 0 to 0.99 & $25.0 \%$ \\
1.0 to 0.99 & $29.1 \%$ \\
2.0 to 2.99 & $24.0 \%$ \\
3.0 to 3.99 & $12.5 \%$ \\
4.0 to 4.99 & $5.2 \%$ \\
5.0 to 5.99 & $2.1 \%$ \\
6.0 to 6.99 & $2.1 \%$ \\
\hline
\end{tabular}


Binkhorst based on biometric data gave results which are clinically valuable.

The postoperative refraction following implantation of an uncalculated 'standard' power IOL is unpredictable, and with a possible change in refraction of over 6 dioptres some problems may be expected from anisometropia when the fellow eye has useful vision. This study shows that the 'standard' power IOL cannot be relied upon to reproduce the preoperative refraction, as has been suggested. This finding is in accord with the results of a study which showed that emmetropic eyes are not identical 'normal' eyes but eyes with different axial lengths and corneal and lens powers which have combined to give emmetropia. ${ }^{9}$ Ametropic eyes can be expected to have achieved their refractions similarly by different combinations of optical factors and to show different optical results on removal of the natural lens. These different biometric factors must be taken into account in the selection of IOL power for controlled postoperative ametropia.
I thank the Department of Medical Illustration at St James's University Hospital, Leeds, for the preparation of illustrations.

\section{References}

1 Roper-Hall MJ. Cataract surgery. Br Med J 1982; 285: 2-3.

2 Kraff MC. Sanders DR, Lieberman HL. Determination of intraocular lens power: a comparison with and without ultrasound. Ophthalmic Surg 1978; 9: 81-4.

3 Clevenger $C E$. Clinical prediction versus ultrasound measurement of IOL power. Am Intra-Ocular Implant Soc J 1978; 4: 222-4.

4 Maloney WF, Kratz RP, Mazzocco TR, Davidson B. Posterior chamber intraocular lens power calculation in 441 cases. Am IntraOcular Implant Soc J 1979; 5: 349-50.

5 Johns GE. Clinical evaluation of the DBR A-scan unit. Am IntraOcular Implant Soc J 1979; 5: 213-6.

6 Hillman JS. Intraocular lens power calculation for emmetropia: a clinical study. Br J Ophthalmol 1982; 66: 53-6.

7 Binkhorst RD. Pitfalls in the determination of intraocular lens power without ultrasound. Ophthalmic Surg 1976; 7: 69-82.

8 Hillman JS. The computer calculation of intraocular lens power: a clinical study. Trans Ophthalmol Soc UK 1980; 100: 222-8.

9 Hillman JS. The selection of intraocular lens power by calculation and by reference to the refraction-a clinical study. Trans Ophthalmol Soc UK in press. 